\title{
P99 WORKING ALONE, IN THE SAFETY ZONE!
}

Helen Birch, Kate Edmondson, Jane Stafford Queenscourt Hospice, Southport, England

10.1136/bmjspcare-2011-000105.99

Background A hospice at home service was set up 2 years ago to work alongside existing primary care services to fill identified gaps in care for patients nearing the end of life whose preferred place of care is home. Part of the service is a $24 \mathrm{~h} 7$ day a week sitting service predominately delivered by trained healthcare assistants (HCA's) plus Bank RN and HCA's.

Aims Staff follow the care plans of the district nurse who undertake all patient assessments. However the personal safety of the staff (lone workers) is the responsibility of the Hospice. A simple daily risk assessment to ensure continuous attention to safety was required.

Method A comprehensive risk assessment checklist which covers all aspects of safety and security is quickly completed by staff at each shift in the patient's home. Completed checklists are checked by the Co-ordinator after every shift with identified risks immediately addressed prior to the next staff member visiting that patient. This complements other staff safety measures: Lone Worker Policy, provision of personal tracker badges and mobile phones.

Results For each of the 4000 patient sits provided since the service began, a checklist has been completed. Recurring themes identified excessive smoking, threatening dogs and 'difficult to find' home addresses as key areas of risk. Expected risk, that is, insecure environment and difficult family dynamics, are hardly featured. A recent survey demonstrated that hospice at home staff felt robust systems were in place to minimise risks to their safety as lone workers in the community.

This poster describes the setting up of the risk assessment system, the checklist, results of the staff survey, and themes emerging from completed checklists. 